\title{
Palladium-Catalyzed Synthesis of Cyclopentane-Fused
}

\section{Benzocyclobutenes via Tandem Directed Carbopalladation/C-H Bond}

\section{Functionalization}

Myra Beaudoin Bertrand and John P. Wolfe*

Department of Chemistry, University of Michigan, 930 N. University Avenue, Ann Arbor, Michigan

48109-1055

\section{Supporting Information}

Experimental procedures and characterization data for new compounds and x-ray crystal structure of 6 (34 pages).

General: All reactions were carried out under an argon or nitrogen atmosphere in oven- or flame-dried glassware. $\mathrm{Pd}(\mathrm{OAc})_{2}$, DPE-phos, anhydrous dioxane, and all starting materials were obtained from commercial sources and used without further purification except 4-bromobenzyl acetate, ${ }^{1}$ and 2cyclopent-2-enylethyl carbamic acid tert-butyl ester, ${ }^{2}$ which were prepared according to published procedures. Ratios of regioisomers and/or diastereomers were determined by ${ }^{1} \mathrm{H}$ NMR and/or capillary $\mathrm{GC}$ analysis of crude reaction mixtures. Yields refer to isolated yields of compounds estimated to be $\geq 95 \%$ pure as determined by ${ }^{1} \mathrm{H}$ NMR, GC, and/or combustion analysis. The yields reported in the supporting information describe the result of a single experiment, whereas the yields reported in Table 1 and eq 2-5 are average yields of two or more experiments. Thus, the yields reported in the supporting information may differ from those shown in Table 1 and eq 2-5. The stereochemistry of 6 was assigned by $x$-ray crystallographic analysis. The stereochemistry of the other benzocyclobutene products was assigned based on analogy to 6 . 
Synthesis of Benzocyclobutene Derivatives and Cyclized Products via Coupling with Aryl Bromides (Equations 2-5 and Table 1)

General Procedure. A flame-dried Schlenk tube equipped with a magnetic stirbar was cooled under a stream of nitrogen and charged with the aryl bromide (1.2 equiv), $\mathrm{Pd}(\mathrm{OAc})_{2}(4 \mathrm{~mol} \%)$, dpe-phos $(8$ mol \%) and $\mathrm{Cs}_{2} \mathrm{CO}_{3}$ (2.3 equiv). The tube was purged with nitrogen and a solution of the $N$-protected amine substrate 3 (1.0 equiv) in dioxane $(4 \mathrm{~mL} / \mathrm{mmol} \mathrm{3})$ was then added via syringe. The resulting mixture was heated to $100{ }^{\circ} \mathrm{C}$ with stirring until the starting material had been consumed as determined by GC analysis. The reaction mixture was cooled to room temperature and saturated aqueous $\mathrm{NH}_{4} \mathrm{Cl}(1$ $\mathrm{mL})$ and ethyl acetate $(1 \mathrm{~mL})$ were added. The layers were separated, the aqueous layer was extracted with ethyl acetate $(3 \times 5 \mathrm{~mL})$, and the combined organic layers were dried over $\mathrm{Na}_{2} \mathrm{SO}_{4}$, filtered, and concentrated in vacuo. The crude product was then purified by flash chromatography on silica gel. All reactions provided the benzocyclobutene derivatives with $>20: 1 \mathrm{dr}$.

( \pm )-(3aR,6S,6aS)-6-Biphenyl-4-yl-hexahydrocyclopenta[b]pyrrole-1-carboxylic acid tert-butyl ester (5). ${ }^{2}$ The general procedure was employed for the reaction of 4-bromobiphenyl (140 mg, 0.60 mmol) with $3(106 \mathrm{mg}, 0.50 \mathrm{mmol})$ except that $\mathrm{NaO} t \mathrm{Bu}(111 \mathrm{mg}, 1.15 \mathrm{mmol})$ was used as a base instead of $\mathrm{Cs}_{2} \mathrm{CO}_{3}$. This procedure afforded $92 \mathrm{mg}(51 \%)$ of the title compound as a white solid, m.p. 126-128 ${ }^{\circ} \mathrm{C}$. This compound was found to exist as a 2:1 mixture of rotamers as judged by ${ }^{1} \mathrm{H}$ NMR analysis. ${ }^{1} \mathrm{H}$ NMR (400 MHz, $\mathrm{CDCl}_{3}$ ) $\delta 7.58-7.52$ (m, $\left.2 \mathrm{H}\right), 7.53-7.45$ (m, $\left.2 \mathrm{H}\right), 7.42$ (t, J = 7.6 Hz, 2 H), 7.28-7.20 (m, 3 H), 4.58-4.48 (m, 0.3 H), 4.43-4.34 (m, 0.7 H), 3.86-3.75 (m, 0.7 H), 3.57-3.45 (m, 0.3 H), 3.39-3.19 (m, 1.3 H), 3.12-3.01 (m, 0.7 H), 3.00-2.81 (m, 1 H), 2.16-2.02 (m, $1 \mathrm{H}), 2.01-$ 1.77 (m, $3 \mathrm{H}), 1.76-1.62$ (m, $2 \mathrm{H}), 1.21-0.93$ (m, $9 \mathrm{H})$.

\section{$( \pm)-(1 R, 3 a R, 7 b S)-2-[6-P h e n y l-2,3,3 a, 7 b-t e t r a h y d r o-1 H$-cyclopenta[3,4]cyclobuta[1,2]benzen-1-}

yl]ethyl-carbamic acid tert-butyl ester (6, Table 1, Entry 2). The general procedure was employed for the reaction of 4-bromobiphenyl $(70 \mathrm{mg}, 0.30 \mathrm{mmol})$ with $3(53 \mathrm{mg}, 0.25 \mathrm{mmol})$. This procedure afforded $71 \mathrm{mg}(75 \%)$ of the title compound as a white solid, m.p. $125-127{ }^{\circ} \mathrm{C} .{ }^{1} \mathrm{H} \mathrm{NMR}(400 \mathrm{MHz}$, $\left.\mathrm{CDCl}_{3}\right) \delta 7.59-7.53(\mathrm{~m}, 2 \mathrm{H}), 7.46-7.38(\mathrm{~m}, 3 \mathrm{H}), 7.34-7.27(\mathrm{~m}, 2 \mathrm{H}), 7.07(\mathrm{~d}, J=7.6 \mathrm{~Hz}, 1 \mathrm{H}), 4.56$ (s, br, $1 \mathrm{H}), 3.85$ (d, J = 4.6 Hz, $2 \mathrm{H}), 3.41-3.28$ (m, $1 \mathrm{H}), 3.29-3.17$ (m, $1 \mathrm{H}), 1.95-1.83$ (m, $2 \mathrm{H})$, 1.81-1.59 (m, $4 \mathrm{H}), 1.46$ (s, $9 \mathrm{H}), 1.13-1.02(\mathrm{~m}, 1 \mathrm{H}) ;{ }^{13} \mathrm{C} \mathrm{NMR}\left(100 \mathrm{MHz}, \mathrm{CDCl}_{3}\right) \delta 155.9,146.3$, $144.4,142.2$, 140.4, 128.6, 127.2, 126.8, 122.2, 121.8, 79.0, 50.7, 47.6, 40.2, 38.6, 32.1, 30.2, 28.7, 
28.4 (two aromatic carbons are incidentally equivalent); IR (film) $1700 \mathrm{~cm}^{-1}$. MS (ESI): 386.2092 (386.2096 calculated for $\mathrm{C}_{24} \mathrm{H}_{29} \mathrm{NO}_{2}, \mathrm{M}+\mathrm{Na}^{+}$).

$( \pm)-(1 S, 3 R)-2-[3-B i p h e n y l-4-y l-c y c l o p e n t y l)-e t h y l]$-carbamic acid tert-butyl ester $(7, \quad \mathrm{Ar}=p$ biphenyl). This compound was isolated as a side-product formed in the reaction of $\mathbf{3}$ with 4 bromobiphenyl described above (10 mg, $5 \%$ yield, ca. 90\% purity). ${ }^{1} \mathrm{H}$ NMR (500 $\mathrm{MHz}, \mathrm{CDCl}_{3}$ )

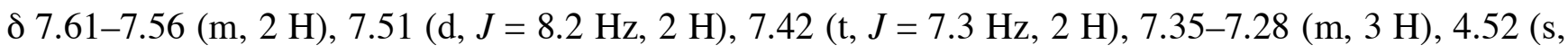
br, $1 \mathrm{H}), 3.21-3.04(\mathrm{~m}, 3 \mathrm{H}), 2.29-2.21(\mathrm{~m}, 1 \mathrm{H}), 2.16-1.92(\mathrm{~m}, 3 \mathrm{H}), 1.77-1.68(\mathrm{~m}, 1 \mathrm{H}), 1.66-1.54$ (m, $3 \mathrm{H}), 1.45$ (s, $9 \mathrm{H}), 1.35-1.24$ (m, $1 \mathrm{H}) ;{ }^{13} \mathrm{C} \mathrm{NMR}\left(100 \mathrm{MHz}, \mathrm{CDCl}_{3}\right) \delta 155.9,145.2,141.1,138.8$, 128.7, 127.4, 127.1, 127.0, 126.8, 79.1, 45.4, 42.1, 39.9, 37.7, 36.7, 33.3, 31.8, 28.4; IR (film) 1699 $\mathrm{cm}^{-1}$. MS (ESI): 388.2247 (388.2252 calculated for $\mathrm{C}_{24} \mathrm{H}_{31} \mathrm{NO}_{2}, \mathrm{M}+\mathrm{Na}^{+}$).

The stereochemistry of $\mathbf{7}$ was determined by ${ }^{1} \mathrm{H}$ NMR nOe analysis as shown below.

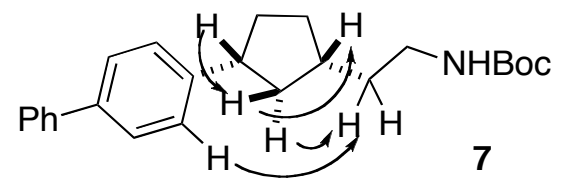

( \pm )-(3R,3aS,7bR)-3-(2-tert-Butoxycarbonylamino-ethyl)-2,3,3a,7b-tetrahydro-1H-

cyclopenta[3,4]cyclobuta[1,2]benzen-5-yl-acetic acid methyl ester (Table 1, Entry 1). The general procedure was employed for the reaction of 4-bromobenzyl acetate $(69 \mathrm{mg}, 0.30 \mathrm{mmol})$ with $\mathbf{3}(53 \mathrm{mg}$, $0.25 \mathrm{mmol})$. This procedure afforded $68 \mathrm{mg}(76 \%)$ of the title compound as a colorless oil. ${ }^{1} \mathrm{H}$ NMR

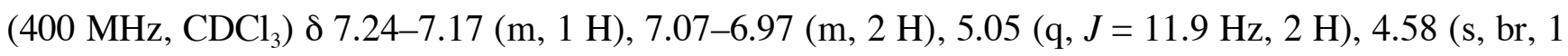
H), $3.79(\mathrm{~d}, J=6.6 \mathrm{~Hz}, 2 \mathrm{H}), 3.35-3.17(\mathrm{~m}, 2 \mathrm{H}), 2.09$ (s, $3 \mathrm{H}), 1.93-1.79(\mathrm{~m}, 2 \mathrm{H}), 1.78-1.51(\mathrm{~m}, 4$ $\mathrm{H}), 1.46(\mathrm{~s}, 9 \mathrm{H}), 1.08-0.94(\mathrm{~m}, 1 \mathrm{H}) ;{ }^{13} \mathrm{C}$ NMR $\left(125 \mathrm{MHz}, \mathrm{CDCl}_{3}\right) \delta 170.9,155.9,147.5,144.3,134.7$, 128.1, 123.6, 121.8, 79.1, 67.1, 50.9, 47.6, 40.3, 38.7, 32.0, 30.1, 28.7, 28.4, 21.1; IR (film) 1740, 1713 $\mathrm{cm}^{-1}$. Anal calcd for $\mathrm{C}_{21} \mathrm{H}_{29} \mathrm{NO}_{4}: \mathrm{C}, 70.17 ; \mathrm{H}, 8.13 ; \mathrm{N}, 3.90$. Found: $\mathrm{C}, 70.31 ; \mathrm{H}, 8.21 ; \mathrm{N}, 3.88$.

( \pm )-(1R,3aR,7bS)-2-[6-Chloro-2,3,3a,7b-tetrahydro-1H-cyclopenta[3,4]cyclobuta[1,2]benzen-1-yl] ethyl-carbamic acid tert-butyl ester (Table 1, Entry 3). The general procedure was employed for the reaction of 4-bromochlorobenzene (115 $\mathrm{mg}, 0.60 \mathrm{mmol})$ with $\mathbf{3}(106 \mathrm{mg}, 0.50 \mathrm{mmol})$. This procedure afforded $120 \mathrm{mg}(71 \%)$ of the title compound as a colorless oil. ${ }^{1} \mathrm{H}$ NMR $\left(500 \mathrm{MHz}, \mathrm{CDCl}_{3}\right) \delta 7.18$ (dd, $J=1.7,7.8 \mathrm{~Hz}, 1 \mathrm{H}), 7.03(\mathrm{~s}, 1 \mathrm{H}), 6.91(\mathrm{~d}, J=7.6 \mathrm{~Hz}, 1 \mathrm{H}), 4.63$ (s, br, $1 \mathrm{H}), 3.76$ (p, $J=3.9 \mathrm{~Hz}$, 2 H), 3.32-3.14 (m, 2 H), 1.92-1.77 (m, 2 H), 1.72-1.63 (m, 2 H), 1.64-1.51 (m, 2 H), 1.46 (s, 9 H), 
1.04-0.94 (m, $1 \mathrm{H}) ;{ }^{13} \mathrm{C}$ NMR (125 MHz, $\left.\mathrm{CDCl}_{3}\right) \delta 155.9,145.2,132.7,127.9,123.7,123.1,79.1$, 50.8, 47.3, 40.1, 38.6, 31.9, 30.0, 28.6, 28.4 (two aromatic carbons are incidentally equivalent); IR (film) $1695 \mathrm{~cm}^{-1}$. Anal calcd for $\mathrm{C}_{18} \mathrm{H}_{24} \mathrm{ClNO}_{2}$ : C, 67.17; H, 7.52; N, 4.35. Found: C, 67.10; H, 7.55; N, 4.38.

\section{$( \pm)-(1 R, 3 a R, 7 b S)-2-6-M e t h o x y-2,3,3 a, 7 b-t e t r a h y d r o-1 H$-cyclopenta[3,4]cyclobuta[1,2]benzen-1-}

yl]ethyl-carbamic acid tert-butyl ester (Table 1, Entry 4). The general procedure was employed for the reaction of 4-bromoanisole $(113 \mathrm{mg}, 0.60 \mathrm{mmol})$ with $3(106 \mathrm{mg}, 0.50 \mathrm{mmol})$. This procedure afforded $98 \mathrm{mg}(62 \%)$ of the title compound as a colorless oil. ${ }^{1} \mathrm{H}$ NMR $\left(500 \mathrm{MHz}, \mathrm{CDCl}_{3}\right) \delta 6.90(\mathrm{~d}, J$ $=8.1 \mathrm{~Hz}, 1 \mathrm{H}), 6.75(\mathrm{dd}, J=2.2,8.1 \mathrm{~Hz}, 1 \mathrm{H}), 6.71(\mathrm{~s}, 1 \mathrm{H}), 4.60(\mathrm{~s}, \mathrm{br}, 1 \mathrm{H}), 3.78(\mathrm{~s}, 3 \mathrm{H}), 3.73(\mathrm{~d}, J=$ $6.3 \mathrm{~Hz}, 2 \mathrm{H}), 3.38-3.27$ (m, 1 H), 3.24-3.15 (m, 1 H), 1.89-1.76 (m, 2 H), 1.75-1.62 (m, 2 H), 1.61$1.52(\mathrm{~m}, 2 \mathrm{H}), 1.45$ (s, $9 \mathrm{H}), 1.07-0.96(\mathrm{~m}, 1 \mathrm{H}) ;{ }^{13} \mathrm{C} \mathrm{NMR}\left(125 \mathrm{MHz}, \mathrm{CDCl}_{3}\right) \delta$ 159.4, 155.9, 144.7, 138.8, 122.6, 114.0, 109.3, 79.1, 55.4, 50.2, 47.0, 40.3, 38.6, 32.1, 30.2, 28.9, 28.4; IR (film) $1700 \mathrm{~cm}^{-}$ ${ }^{1}$. Anal calcd for $\mathrm{C}_{19} \mathrm{H}_{27} \mathrm{NO}_{3}: \mathrm{C}, 71.89 ; \mathrm{H}, 8.57 ; \mathrm{N}, 4.41$. Found: C, 72.06; H, 8.59; N, 4.36.

$( \pm)-(1 R, 3 a R, 7 b S)-2-[4-M e t h y l-2,3,3 a, 7 b-t e t r a h y d r o-1 H$-cyclopenta[3,4]cyclobuta[1,2]benzen-1yl]ethyl-carbamic acid tert-butyl ester (Table 1, Entry 5). The general procedure was employed for the reaction of 2-bromotoluene $(103 \mathrm{mg}, 0.60 \mathrm{mmol})$ with $3(106 \mathrm{mg}, 0.50 \mathrm{mmol})$. This procedure afforded $130 \mathrm{mg}(87 \%)$ of the title compound as a colorless oil. ${ }^{1} \mathrm{H}$ NMR $\left(400 \mathrm{MHz}, \mathrm{CDCl}_{3}\right) \delta 7.09(\mathrm{t}$, $J=7.6 \mathrm{~Hz}, 1 \mathrm{H}), 6.98(\mathrm{~d}, J=7.8 \mathrm{~Hz}, 1 \mathrm{H}), 6.83$ (d, J=7.0 Hz, $1 \mathrm{H}), 4.56$ (s, br, 1 H), 3.83-3.72 (m, 2 H), 3.36-3.16 (m, 2 H), 2.17 (s, 3 H), 1.90-1.79 (m, 2 H), 1.75-1.62 (m, 2 H), 1.61-1.51 (m, 2 H), 1.45 $(\mathrm{s}, 9 \mathrm{H}), 1.07-0.95(\mathrm{~m}, 1 \mathrm{H}) ;{ }^{13} \mathrm{C} \mathrm{NMR}\left(100 \mathrm{MHz}, \mathrm{CDCl}_{3}\right) \delta 155.9,145.2,143.5,131.9,128.2,127.3$, $120.5,79.1,50.4,47.0,40.3,38.5,31.9,30.2,28.4,27.6,16.2$; IR (film) $1696 \mathrm{~cm}^{-1}$. Anal calcd for $\mathrm{C}_{19} \mathrm{H}_{27} \mathrm{NO}_{2}$ : C, 75.71; H, 9.03; N, 4.65. Found: C, 75.61; H, 9.04; N, 4.64.

\section{$( \pm)-(1 R, 3 a R, 7 b S)-2-[4-P h e n y l-2,3,3 a, 7 b-t e t r a h y d r o-1 H$-cyclopenta[3,4]cyclobuta[1,2]benzen-1-}

yl]ethyl-carbamic acid tert-butyl ester (Table 1, Entry 6). The general procedure was employed for the reaction of 2-bromobiphenyl $(70 \mathrm{mg}, 0.30 \mathrm{mmol})$ with $3(53 \mathrm{mg}, 0.25 \mathrm{mmol})$. This procedure afforded $68 \mathrm{mg}(75 \%)$ of the title compound as a white solid (m.p. $\left.118-120{ }^{\circ} \mathrm{C}\right) .{ }^{1} \mathrm{H} \mathrm{NMR}(400 \mathrm{MHz}$, $\left.\mathrm{CDCl}_{3}\right) \delta 7.67(\mathrm{~d}, J=7.4 \mathrm{~Hz}, 2 \mathrm{H}), 7.48(\mathrm{~d}, J=8.2 \mathrm{~Hz}, 1 \mathrm{H}), 7.41(\mathrm{t}, J=7.6 \mathrm{~Hz}, 2 \mathrm{H}), 7.33-7.23(\mathrm{~m}, 2$ H), $6.98(\mathrm{~d}, J=7.2 \mathrm{~Hz}, 1 \mathrm{H}), 4.58$ (s, br, $1 \mathrm{H}), 4.18-4.10$ (m, 1 H), 3.89-3.81 (m, 1 H), 3.37-3.17 (m, 2 H), 1.96-1.86 (m, $1 \mathrm{H}), 1.85-1.79(\mathrm{~m}, 1 \mathrm{H}), 1.78-1.70(\mathrm{~m}, 1 \mathrm{H}), 1.69-1.54(\mathrm{~m}, 3 \mathrm{H}), 1.46$ (s, $9 \mathrm{H})$, 
1.09-0.96 (m, $1 \mathrm{H}) ;{ }^{13} \mathrm{C}$ NMR $\left(100 \mathrm{MHz}, \mathrm{CDCl}_{3}\right) \delta 155.9,144.7,143.8,138.0,134.4,128.7,128.0$, 127.2, 126.9, 125.2, 122.2, 79.1, 50.6, 48.9, 40.3, 38.8, 31.8, 30.2, 28.4, 27.7; IR (film) $1699 \mathrm{~cm}^{-1}$. Anal calcd for $\mathrm{C}_{24} \mathrm{H}_{29} \mathrm{NO}_{2}$ : C, 79.30; H, 8.04; N, 3.85. Found: C, 79.01; H, 8.05; N, 3.84 .

\section{$( \pm)-[(1 R, 3 \mathrm{a} R, 7 \mathrm{bS})$-2-[5-Methyl-2,3,3a,7b-tetrahydro-1H-cyclopenta[3,4]cyclobuta[1,2]benzen-1-}

yl]ethyl-carbamic acid tert-butyl ester (Table 1, Entry 7). The general procedure was employed for the reaction of 3-bromotoluene $(52 \mathrm{mg}, 0.30 \mathrm{mmol})$ with $3(53 \mathrm{mg}, 0.25 \mathrm{mmol})$. This procedure afforded $56 \mathrm{mg}(75 \%)$ of the title compound as a colorless oil. ${ }^{1} \mathrm{H}$ NMR $\left(400 \mathrm{MHz}, \mathrm{CDCl}_{3}\right) \delta 6.96(\mathrm{~d}, J$ $=7.6 \mathrm{~Hz}, 1 \mathrm{H}), 6.87(\mathrm{~d}, J=7.2 \mathrm{~Hz}, 1 \mathrm{H}), 6.79(\mathrm{~s}, 1 \mathrm{H}), 4.51(\mathrm{~s}, \mathrm{br}, 1 \mathrm{H}), 3.72(\mathrm{~m}, 2 \mathrm{H}), 3.31-3.11(\mathrm{~m}, 2$ H), 2.28 (s, $3 \mathrm{H}), 1.86-1.73(\mathrm{~m}, 2 \mathrm{H}), 1.71-1.48(\mathrm{~m}, 4 \mathrm{H}), 1.41$ (s, $9 \mathrm{H}), 1.03-0.90(\mathrm{~m}, 1 \mathrm{H}) ;{ }^{13} \mathrm{C}$ NMR $\left(100 \mathrm{MHz}, \mathrm{CDCl}_{3}\right) \delta 155.9,147.3,140.6,137.1,127.7,123.0,122.4,79.1,50.5,47.6,40.3,38.6,32.0$, 30.1, 28.7, 28.4, 22.1; IR (film) $1699 \mathrm{~cm}^{-1}$. Anal calcd for $\mathrm{C}_{19} \mathrm{H}_{27} \mathrm{NO}_{2}$ : C, 75.71; H, 9.03; N, 4.65. Found: C, 75.53; H, 9.02; N, 4.47 .

\section{$( \pm)-(1 R, 3 a R, 7 b S)-2-[5-M e t h o x y-2,3,3 a, 7 b-t e t r a h y d r o-1 H$-cyclopenta[3,4]cyclobuta[1,2]benzen-1-}

yl]ethyl-carbamic acid tert-butyl ester (Table 1, Entry 8). The general procedure was employed for the reaction of 3-bromoanisole $(113 \mathrm{mg}, 0.6 \mathrm{mmol})$ with $3(106 \mathrm{mg}, 0.5 \mathrm{mmol})$. This procedure afforded $107 \mathrm{mg}$ (67\%) of the title compound as an inseparable 2:1 mixture of regioisomers (colorless oil). Data are for the mixture ${ }^{1} \mathrm{H}$ NMR $\left(400 \mathrm{MHz}, \mathrm{CDCl}_{3}\right) \delta 7.19(\mathrm{t}, J=7.6 \mathrm{~Hz}, 0.33 \mathrm{H}), 6.93$ (d, $J=$ $8.0 \mathrm{~Hz}, 0.67 \mathrm{H}), 6.77-6.70(\mathrm{~m}, 1 \mathrm{H}), 6.69-6.63(\mathrm{~m}, 0.33 \mathrm{H}), 6.62-6.58$ (m, $0.67 \mathrm{H}), 5.02$ (s, br, 0.33 H), 4.62 (s, br, $0.67 \mathrm{H}), 3.89-3.83(\mathrm{~m}, 0.33 \mathrm{H}), 3.82-3.68(\mathrm{~m}, 4.67 \mathrm{H}), 3.39-3.14(\mathrm{~m}, 2 \mathrm{H}), 1.89-1.75$ (m, $2 \mathrm{H}), 1.74-1.51(\mathrm{~m}, 4 \mathrm{H}), 1.46(\mathrm{~s}, 9 \mathrm{H}), 1.18-1.07$ (m, 0.33 H), 1.06-0.94 (m, 0.67 H); ${ }^{13} \mathrm{C}$ NMR $\left(100 \mathrm{MHz}, \mathrm{CDCl}_{3}\right) \delta 159.8,156.1,155.9,154.0,149.1,148.0,135.4,129.7,129.2,124.2,114.6,113.4$, 109.4, 107.5, 79.0, 78.7, 55.3, 55.0, 50.0, 49.9, 47.7, 47.1, 40.6, 40.2, 40.0, 38.6, 31.9, 30.5, 30.1, 28.6, 28.43, 28.39, 28.0 (two aliphatic carbons are incidentally equivalent); IR (film) $1699 \mathrm{~cm}^{-1}$. Anal calcd for $\mathrm{C}_{19} \mathrm{H}_{27} \mathrm{NO}_{3}$ : C, 71.89; H, 8.57; N, 4.41. Found: C, 71.99; H, 8.72; N, 4.34.

\section{$( \pm)-(1 R, 3 \mathrm{a} R, 7 \mathrm{bS})-2-[5-T r i f l u o r o m e t h y l-2,3,3 \mathrm{a}, 7 \mathrm{~b}-$ tetrahydro-1 $H$ -}

cyclopenta[3,4]cyclobuta[1,2]benzen-1-yl]ethyl-carbamic acid tert-butyl ester (11, Equation 4). The general procedure was employed for the reaction of 3-bromobenzotrifluoride (270 $\mathrm{mg}, 1.2 \mathrm{mmol}$ ) with 3 (212 mg, $1.0 \mathrm{mmol})$. This procedure afforded $84 \mathrm{mg}$ (24\%) of the title compound as a colorless oil. ${ }^{1} \mathrm{H}$ NMR (500 MHz, $\left.\mathrm{CDCl}_{3}\right) \delta 7.46(\mathrm{~d}, J=7.6 \mathrm{~Hz}, 1 \mathrm{H}), 7.25(\mathrm{~s}, 1 \mathrm{H}), 7.16(\mathrm{~d}, J=7.3 \mathrm{~Hz}, 1 \mathrm{H})$, 
4.60 (s, br, $1 \mathrm{H}), 3.85$ (d, $J=6.3 \mathrm{~Hz}, 2 \mathrm{H}), 3.37-3.27$ (m, $1 \mathrm{H}), 3.26-3.15(\mathrm{~m}, 1 \mathrm{H}), 1.97-1.83(\mathrm{~m}, 2 \mathrm{H})$, 1.76-1.61 (m, $3 \mathrm{H}), 1.60-1.51(\mathrm{~m}, 1 \mathrm{H}), 1.46(\mathrm{~s}, 9 \mathrm{H}), 1.04-0.92(\mathrm{~m}, 1 \mathrm{H}) ;{ }^{13} \mathrm{C} \mathrm{NMR}(125 \mathrm{MHz}$, $\left.\mathrm{CDCl}_{3}\right) \delta 155.9,148.2,147.7,129.8(\mathrm{q}, J=31.8 \mathrm{~Hz}), 124.6(\mathrm{q}, J=272.5 \mathrm{~Hz}), 124.3,123.5,118.7$, 79.2, 51.2, 47.8, 40.2, 38.6, 32.0, 30.1, 28.6, 28.4; IR (film) $1699 \mathrm{~cm}^{-1}$. Anal calcd for $\mathrm{C}_{19} \mathrm{H}_{24} \mathrm{~F}_{3} \mathrm{NO}_{2}: \mathrm{C}_{\text {, }}$ 64.21; H, 6.81; N, 3.94. Found: C, 64.19; H, 7.00; N, 3.94.

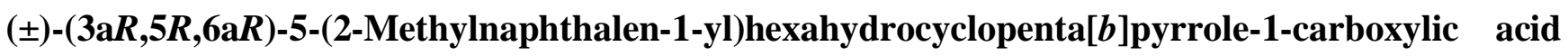
tert-butyl ester (12). The general procedure was employed for the reaction of 1-bromo-2methylnaphthalene $(67 \mathrm{mg}, 0.30 \mathrm{mmol})$ with $3(53 \mathrm{mg}, 0.25 \mathrm{mmol})$. This procedure afforded $23 \mathrm{mg}$ (26\%) of the title compound as a colorless oil and as a $\sim 2: 1$ mixture of rotamers. ${ }^{1} \mathrm{H}$ NMR $(500 \mathrm{MHz}$, $\left.\mathrm{CDCl}_{3}\right) \delta 8.12(\mathrm{~d}, J=8.3 \mathrm{~Hz}, 1 \mathrm{H}), 7.79(\mathrm{~d}, J=7.8 \mathrm{~Hz}, 1 \mathrm{H}), 7.64-7.57(\mathrm{~m}, 1 \mathrm{H}), 7.48-7.34(\mathrm{~m}, 2 \mathrm{H})$, 7.30-7.23 (m, 1 H), 4.36-4.21 (m, 1 H), 4.02-3.87 (m, 1 H), 3.84-3.77 (m, 0.7 H), 3.74-3.67 (m, 0.3 H), 3.65-3.55 (m, $1 \mathrm{H}), 2.92-2.82(\mathrm{~m}, 1 \mathrm{H}), 2.57-2.42(\mathrm{~m}, 5 \mathrm{H}), 2.30-2.16(\mathrm{~m}, 1 \mathrm{H}), 2.10-1.97$ (m, 2 $\mathrm{H}), 1.87-1.79(\mathrm{~m}, 1 \mathrm{H}), 1.54-1.36(\mathrm{~m}, 9 \mathrm{H}) ;{ }^{13} \mathrm{C} \mathrm{NMR}\left(125 \mathrm{MHz}, \mathrm{CDCl}_{3}\right) \delta 154.5,135.1,133.6,133.4$, $131.7,129.7,129.2,126.8,126.7,125.3,124.8,124.3,124.2,124.1,79.1,63.3,62.9,45.6,45.1,44.8$, 44.0, 41.3, 39.5, 38.1, 36.3, 29.4, 28.8, 28.6, 28.5, 21.8; IR (film) $1692 \mathrm{~cm}^{-1}$. MS (ESI): 374.2087 (374.2096 calculated for $\mathrm{C}_{23} \mathrm{H}_{29} \mathrm{NO}_{2}, \mathrm{M}+\mathrm{Na}^{+}$).
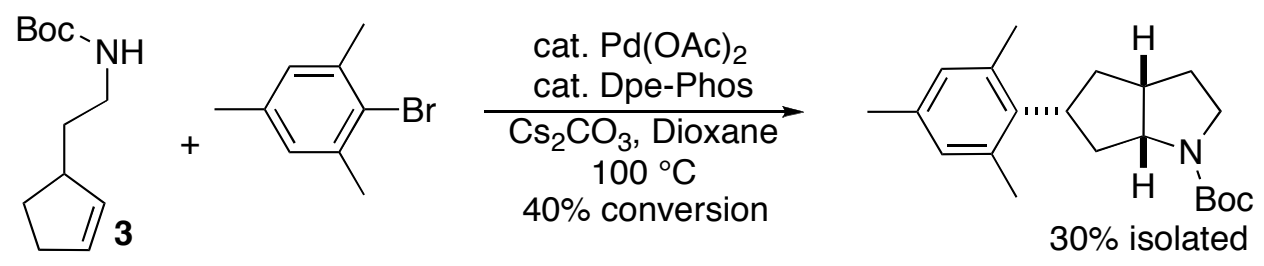

$( \pm)-(3 a R, 5 R, 6 a R)-5-(2,4,6-$ Trimethylphenyl)hexahydrocyclopenta[b]pyrrole-1-carboxylic acid tert-butyl ester (Described in footnote 9). The general procedure was employed for the reaction of 2bromomesitylene $(60 \mathrm{mg}, 0.30 \mathrm{mmol})$ with $3(53 \mathrm{mg}, 0.25 \mathrm{mmol})$. This procedure afforded $25 \mathrm{mg}$ (30\%) of the title compound as a colorless oil and as a $\sim 2: 1$ mixture of rotamers. ${ }^{1} \mathrm{H}$ NMR $(500 \mathrm{MHz}$, $\left.\mathrm{CDCl}_{3}\right) \delta 6.86-6.78(\mathrm{~m}, 2 \mathrm{H}), 4.23-4.10(\mathrm{~m}, 1 \mathrm{H}), 3.73-3.65$ (m, 0.7 H), 3.62-3.55 (m, 0.3 H), 3.523.36 (m, 2 H), 2.79-2.67 (m, 1 H), 2.42-2.29 (m, 7 H), 2.23 (s, 3 H), 2.11-1.82 (m, 4 H), 1.78-1.70 $(\mathrm{m}, 1 \mathrm{H}), 1.52-1.39(\mathrm{~m}, 9 \mathrm{H}) ;{ }^{13} \mathrm{C} \mathrm{NMR}\left(125 \mathrm{MHz}, \mathrm{CDCl}_{3}\right) \delta 154.4,136.7,135.4,135.3,130.3,130.2$, 79.0, 62.6, 62.3, 45.5, 44.9, 44.3, 43.3, 41.4, 39.0, 37.4, 35.1, 29.7, 28.9, 28.5, 21.4, 21.2, 20.6; IR (film) $1695 \mathrm{~cm}^{-1}$. MS (ESI): 352.2249 (352.2252 calculated for $\mathrm{C}_{21} \mathrm{H}_{31} \mathrm{NO}_{2}, \mathrm{M}+\mathrm{Na}^{+}$). 


\section{References}

(1) Bertrand, M. B.; Leathen, M. L.; Wolfe, J. P. Org. Lett. 2007, 9, 457-460.

(2) Bertrand, M. B.; Wolfe, J. P. Tetrahedron, 2005, 61, 6447-6459. 